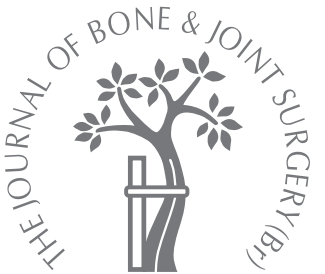

F. Canavese,

S. Gupta,

J. I. Krajbich,

K. M. Emara

From the Shriners

Hospital for

Children, Portland,

USA

\title{
Vacuum-assisted closure for deep infection after spinal instrumentation for scoliosis
}

\begin{abstract}
Our aim was to review the efficacy of the wound vacuum-assisted closure (VAC) system in the treatment of deep infection after extensive instrumentation and fusion for spinal deformity in children and adolescents.

A total of 14 patients with early deep spinal infection were treated using this technique. Of these, 12 had neuromuscular or syndromic problems. Clinical and laboratory data were reviewed. The mean follow-up was 44 months (24 to 72). All wounds healed. Two patients required plastic surgery to speed up the process. In no patient was the hardware removed and there was no loss of correction or recurrent infection.

We believe that the wound VAC system is a useful tool in the armamentarium of the spinal surgeon dealing with patients susceptible to wound infections, especially those with neuromuscular diseases. It allows for the retention of the instrumentation and the maintenance of spinal correction. It is reliable and easy to use.
\end{abstract}

Deep infection after instrumented fusion in the management of scoliosis is uncommon. However, when it does occur, it can result in considerable morbidity, cost, and compromise of correction. As surgical and peri-operative techniques have improved, more severely involved children with complex neuromuscular deformities and considerable co-morbidities are now thought to be candidates for extensive surgery for spinal deformity. Rates of surgical infection of up to $20 \%$ for this type of patient with scoliosis have been reported. ${ }^{1-4}$ Various treatment protocols for debridement, soft-tissue management and antibiotic therapy have been recommended with mixed results. The use of the wound vacuum-assisted closure (VAC) system (KCI Inc, San Antonio, Texas) has gained increasing popularity in the management of acute, subacute and chronic wounds. The controlled application of subatmospheric pressure helps the formation of granulation tissue, assists debridement of necrotic tissue and acts as a sterile barrier. Increasing use of the VAC system for complex soft-tissue injuries ${ }^{5-9}$ has generally resulted in accelerated wound healing compared with traditional methods. Our aim was to review the use of the VAC system in the management of early-onset deep infection after spinal instrumentation.

\section{Patients and Methods}

We carried out a retrospective review of the notes and radiographs of all cases of early- onset deep infection of the spine which had occurred between March 1997 and March 2005, and identified 14 such patients who had been treated by the VAC system. There were ten girls and four boys with a mean age of 13.4 years (3 to 19). The underlying diagnoses are shown in Table I. Patients with only superficial infections were not treated by the VAC system and were, therefore, excluded.

Application of the vacuum-assisted closure system. The system consists of polyurethane ether foam sponge with open pores, $400 \mathrm{~mm}$ to $600 \mathrm{~mm}$ in size, a connecting tube and a plastic sealant. After thorough lavage and removal of all macroscopic contamination, devitalised tissue and loose bone graft, the VAC sponge is cut and fitted into the wound. The plastic sealant is used to cover the sponge and is applied several centimetres beyond the margins of the wound to create an air-tight seal. A cruciate incision is made in the plastic sealant covering the sponge through which a suction tube is inserted and fixed. The tubing is connected to a negativepressure device. The sponge is compressed at subatmospheric pressure $(-125 \mathrm{mmHg})$, continuously or intermittently. The 'controlled negative pressure' is used to evacuate oedema from the wound, increase blood-flow, decrease bacterial load and increase the formation of granulation tissue. The system also assists the debridement of necrotic tissue and acts as a sterile barrier. 
Table I. Details of the 14 patients

\begin{tabular}{|c|c|c|c|c|}
\hline Case & Gender & Diagnosis & VAC $^{*}$ (days) & $\begin{array}{l}\text { Follow-up } \\
\text { (mths) }\end{array}$ \\
\hline 1 & $M$ & Duchenne's muscular dystrophy & 5 & 72 \\
\hline 2 & $\mathrm{M}$ & Cerebral palsy, spastic quadriplegia & 21 & 55 \\
\hline 3 & $\mathrm{~F}$ & Velocardiofacial syndrome & 8 & 33 \\
\hline 4 & $\mathrm{~F}$ & Prader-Willi syndrome & 7 & 63 \\
\hline 5 & $\mathrm{~F}$ & Lennox-Gastaut syndrome & 14 & 59 \\
\hline 6 & $\mathrm{~F}$ & T5 myelodysplasia & 42 & 47 \\
\hline 7 & $\mathrm{~F}$ & Cerebral palsy, spastic quadriplegia & 22 & 55 \\
\hline 8 & $\mathrm{~F}$ & Cerebral palsy, spastic quadriplegia & 41 & 40 \\
\hline 9 & $\mathrm{~F}$ & Infantile scoliosis & 14 & 36 \\
\hline 10 & M & L3 myelodysplasia & 28 & 52 \\
\hline 11 & $\mathrm{~F}$ & L1 myelodysplasia & 38 & 24 \\
\hline 12 & $\mathrm{~F}$ & Spinal muscular atrophy type 3 & 26 & 24 \\
\hline 13 & $\mathrm{~F}$ & Idiopathic & 19 & 28 \\
\hline 14 & M & Spinal muscular atrophy type 2 & 14 & 25 \\
\hline
\end{tabular}

* VAC, vacuum-assisted closure

Table II. The type of surgery performed and the type of instrumentation implanted in the 14 patients

\begin{tabular}{cllll}
\hline Case & Aetiology & Anatomical site & Type of surgery & Type of instrumentation $^{\dagger}$ \\
\hline 1 & Paralytic & Thoracolumbar & PSF & UR, T2 to pelvis \\
2 & Neuromuscular & Thoracolumbar & AR + PSF & UR, T1 to L3 \\
3 & Neuromuscular & Thoracolumbar & PSF & SI, T9 to L3 \\
4 & Neuromuscular & Kyphosis & AR + PSF & UR, T4 to L3 \\
5 & Paralytic & Thoracolumbar & PSF & LR, T1 to pelvis \\
6 & Paralytic & Thoracolumbar & PSF & LR, T1 to pelvis \\
7 & Neuromuscular & Thoracolumbar & AR + PSF & UR, T2 to pelvis \\
8 & Neuromuscular & Thoracolumbar & PSF & UR, T3 to pelvis \\
9 & Idiopathic & Thoracolumbar & PSF & SI, T4 to L3 \\
10 & Neuromuscular & Thoracic & AR + PSF & SI, T6 to T12 \\
11 & Neuromuscular & Thoracolumbar & AR + PSF & SI, T8 to L3 \\
12 & Neuromuscular & Thoracolumbar & AR + PSF & LR, T2 to pelvis \\
13 & Idiopathic & Thoracolumbar & PSF & SI, T3 to T11 \\
14 & Neuromuscular & Thoracolumbar & PSF & LR, T2 to pelvis \\
\hline * PSF, posterior spinal fusion; AR, anterior release &
\end{tabular}

† UR, unit rod; SI, segmental instrumentation; LR, Luque rods

The decision to use the VAC system was at the discretion of the surgeon (JIK) and was based on the macroscopic appearance of the wound and the underlying diagnosis. Most of our patients had cognitive and functional problems (Table I) and some were overweight. Those with myelodysplasia and spastic quadriplegia, with paralytic or poor bladder control typically suffered from infections of the urinary tract.

Thoracolumbar curvatures were present in 12 patients, with one having an isolated thoracic curvature and one a kyphosis (Table II). The spinal instrumentation used varied according to the underlying pathology. Standard segmental instrumentation using dual rods, hooks and pedicle screws was used for non-neuromuscular curves and segmental instrumentation into the pelvis for most of the neuromuscular curves (Unit rod or Luque rods; Table II).

All the patients were evaluated for eradication of infection using clinical, radiological and laboratory indicators such as the presence of spinal pseudarthrosis, the level of C- reactive protein (CRP) and the white cell count (WCC), the need for additional surgery after application of the VAC system, any loss of correction, and loosening or removal of hardware. Intra-operative debridement involved thorough lavage and removal of all macroscopic contamination, devitalised tissue and loose bone graft (Figs 1 and 2). No attempt was made to remove graft which was partially or fully fused. Intra-operative specimens for bacteriological culture were obtained before application of the VAC system.

\section{Results}

Combined anterior release and posterior fusion was performed in six patients, and posterior fusion alone in eight patients (Table II). Allograft was used in 13 patients. The mean time for surgery was 417 minutes (124 to 715). Both superficial and deep drains were inserted at the primary procedure in ten patients.

All 14 patients presented with a discharge from part of the wound; only eight had constitutional symptoms. No 


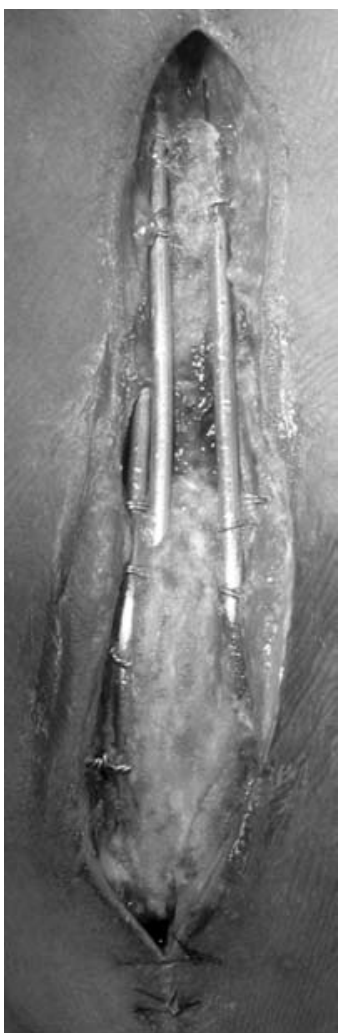

Fig. 1

Photograph of a wound before application of the vacuumassisted closure system. The bone graft is still in place, as well as the hardware.

patient had exposed hardware at the time of presentation. The infection presented at a mean of 16 days after surgery (7 to 46). All had evaluation of inflammatory markers (CRP and WCC), intra-operative bacteriological culture, surgical debridement and application of the VAC system. In two patients, antibiotics had been administered before presentation to our institution. All were started on appropriate broad-spectrum antibiotics until sensitivities were available, when treatment was changed to a more specific antibiotic.

The VAC system was applied at the intial surgical debridement in ten patients. Debridement was undertaken in two patients before application, and in two the VAC was applied as the third procedure. These patients were treated at the beginning of our experience. The mean number of procedures required for the change of the VAC was 3.4 (1 to 10$)$. The mean length of application was 21 days ( 5 to 42 ) and the VAC dressing was changed twice weekly on average. The mean follow-up was 44 months ( 24 to 72 ).

All patients received antibiotics (intravenously and/or orally) for at least six weeks based on the results of the wound-culture sensitivities. They were maintained on anti-

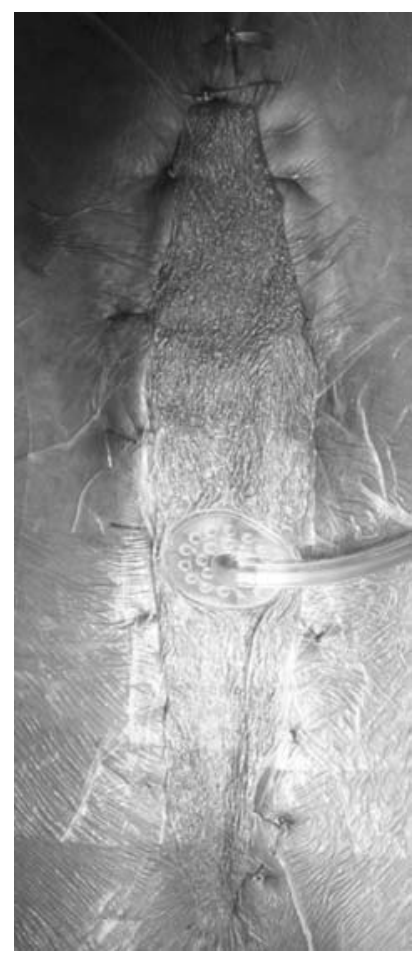

Fig. 2

Photograph of a wound after the application of the vacuum-assisted system. biotics for the length of time that the wound was open. The mean time for the administration of intravenous antibiotics was six weeks (1 to 12 ). This was followed by oral antibiotics for a mean of six months (4 weeks to 12 months). The organisms cultured included Staphylococcus aureus in five patients, Escherichia coli combined with Pseudomonas aeruginosa in two, Streptococcus pneumoniae in one, E. coli in one, Enterobacter aerogenus in one, P. aeruginosa in one, and Enterobacter cloacae in one. In two patients, there was no formal growth. It was possible to discharge five patients from hospital as outpatients with continued application of the VAC system. Dressing changes were performed twice weekly as an outpatient procedure. The remaining patients were treated as in-patients because of the problems of twice-weekly long-distance travel to our institution. If travel had been easy, most could have been treated as outpatients after the first two or three dressing changes.

The wounds were regularly reviewed and eventually healed by secondary intention. No complications occurred related to the use of the system. Although technical problems such as the blockage of tubes, leaks or breakage of 
seals are possible ${ }^{8}$ none of our patients experienced these since most remained in hospital during the period of treatment. Two patients required plastic surgery for definitive closure once the wound was clear of infection. We elected to do this to speed up the process of closure and to provide cover for the healthy granulation tissue rather than wait for natural epithelialisation. Both patients made an uncomplicated recovery. In the remaining 12 patients, we let the wounds heal naturally. No patient required removal of hardware either initially or during follow-up. As a result, there was no loss of correction of the spinal deformity. No patient required further surgery either for recurrent infection, pseudarthrosis or problems with hardware. Of the 14 patients, four had a normal CRP when they presented with infection and it remained normal after treatment. Three patients had a normal WCC when they presented with infection and it remained normal after treatment. The mean CRP and WCC at the time of presentation were $6.7 \mathrm{mg} / \mathrm{l}$ (1.4 to 17.4$)$ and $11.3 \times 10^{9}$ cells/l $(6.4$ to 16.5$)$, respectively, which decreased to $0.7 \mathrm{mg} / \mathrm{l}(0.3$ to 0.9$)$ and $6.4 \times$ $10^{9}$ cells/l (3.8 to 12$)$, respectively, after application of the VAC system and treatment with antibiotics. In one patient, the CRP remained slightly elevated after the treatment at $2.3 \mathrm{mg} / \mathrm{l}$. This patient had a myelomeningocele with urinary-tract problems unrelated to the spinal surgery.

\section{Discussion}

Vacuum-assisted closure is a relatively new technique for promoting the healing of infected wounds resistant to treatment by established methods. ${ }^{5}$ In the literature, the rate of spinal infections has been reported to increase with the complexity of the procedure, ranging between $1.9 \%$ and $20 \%{ }^{7,10,11}$

Since its introduction in $1997,{ }^{5}$ the VAC system has been widely used in the adult population. A decrease in wound complications, improved healing times and reduced overall morbidity have been reported but there are limited data on its use in children. ${ }^{6,9,12}$

Mooney et $\mathrm{al}^{6}$ reported successful results in children with extensive and complex soft-tissue wounds. There were fewer dressing changes and potentially less extensive skin cover procedures. With advances in anaesthesia, intensive care and medical therapy, patients with more complex comorbidities are increasingly being considered for extensive spinal intervention. One of the aims of the technique in the child is to reduce the need for further complex soft-tissue procedures, removal of hardware with consequent loss of correction, and pseudarthrosis. The use of the VAC system is particularly appealing in patients with multiple comorbidities since it may avoid the need for complex plastic surgery and later re-operation.

Mehbod et $\mathrm{al}^{7}$ reported successful results using this system in the management of deep spinal infection in adults. They studied a heterogeneous group of 20 patients, of whom 16 had early- and four late-onset infections. Their patients had many risk factors ${ }^{13}$ for infection including age, diabetes, smoking, tumour or immunodeficiency with a short mean follow-up of ten months. In our study, the population was children or adolescents with severe deformity of the spine who developed early-onset deep infection after spinal instrumentation. In 12 of the 14, there were considerable neurological problems such as myelodysplasia or spinal muscular atrophy. Our mean follow-up was 44 months (24 to 72 ). Mehbod et $\mathrm{al}^{7}$ reported that approximately 2.2 procedures ( 2 to 3 ) were required to obtain definitive closure, which occurred at a mean of seven days (5 to 14 ) after initial placement. However, only eight patients had placement of the VAC system as the first procedure and 12 required two or more debridements under general anaesthesia before the application of the system. Our patients had more levels fused than those reported by Mehbod et $\mathrm{al}^{7}$ (10 vs 4) and ten of our 14 patients had application of the system after the initial debridement.

Picada et al, ${ }^{10}$ using a protocol of aggressive debridement and delayed primary or secondary closure, achieved eradication of infection in 24 of 26 adult patients with deep infection after lumbosacral fusion. They had to remove the hardware in seven patients, in two of whom this was related to infection and in five to 'localised discomfort'. None of our patients required removal of hardware and all infections were successfully managed by the VAC system.

Our study has shown that a higher mean number of procedures and a longer time are required for definitive wound closure compared with these other series.

We believe that this is because of the co-morbidities such as neuromuscular and syndromic disorders and the more extensive spinal surgery in the children and adolescents we treated. Most of our patients were unable to walk, with limited ability for self-care. Furthermore, because of the nature of the spinal deformity, with instrumentation generally from the upper thoracic spine to the pelvis, our patients required longer incisions and a higher number of levels fused, compared with adult patients. This predisposes to a higher rate of wound complications..$^{2-4,7,10,11}$

Persisting deep infection often necessitates the removal of hardware. ${ }^{14,15}$ This presents a major problem in the patient with scoliosis, with the potential for loss of correction. Studies in adults ${ }^{16}$ have shown rates of removal of hardware in patients with deep spinal infection of up to $35 \%$. Although a more recent study ${ }^{17}$ has shown a considerably lower rate, there have been few studies of early post-operative deep spinal infection after surgery for scoliosis in children.

Richards et $\mathrm{al}^{14}$ reported removal of hardware in all ten patients in their series of deep infections after scoliosis surgery. However, the mean time to presentation was 25 months (11 to 45 ) after surgery. In all except two patients, successful arthrodesis had been achieved. Similarly, Soultanis et $\mathrm{al}^{18}$ reported five cases of late deep infection in patients with scoliosis after spinal instrumentation. At the time of debridement, all had achieved a successful arthrodesis, and the instrumentation was removed without com- 
plication. Our study concerned the management of early post-operative infection in which removal of the implant is undesirable since fusion had not been achieved. However, no patient required removal of hardware and there was no loss of correction.

Our experience of this technique in 14 patients suggests that it is useful in the management of early deep infection in children and adolescents after extensive surgery for scoliosis. The frequency of dressing changes (twice a week) is less than with more traditional methods and can be undertaken on an outpatient basis if this is logistically possible for the patient and the family.

No benefits in any form have been received or will be received from a commercial party related directly or indirectly to the subject of this article.

\section{References}

1. Klink BK, Thurman RT, Witpenn GP, Lauerman WC, Coin JE. Muscle flap closure for salvage of complex back wounds. Spine 1994;19:1467-70.

2. Malamo-Lada H, Zarkotou O, Nikolaides N, Kanellopoulou M, Demetriades D. Wound infections following posterior spinal instrumentation for paralytic scoliosis. Clin Microbial Infect 1999:5:135-9.

3. Sriram K, Bobechko WP, Hall JE. Surgical management of spinal deformities in spinal bifida. J Bone Joint Surg [Br] 1972;54-B:666-76.

4. McCarthy JJ, D'Andrea LP, Betz RR, Clements DH. Scoliosis in the child with cerebral palsy. J Am Acad Surg 2006;14:356-75.

5. Argenta LC, Morykwas MJ. Vacuum-assisted wound closure: a new method for wound control and treatment: clinical experience. Ann Plast Surg 1997;38:563-76.
6. Mooney JF 3rd, Argenta LC, Marks MW, Morykwas MJ, DeFranzo AJ. Treatment of soft tissue defects in pediatric patients using the V.A.C. system. Clin Orthop 2000;376:26-31.

7. Mehbod AA, Ogilvie JW, Pinto MR, et al. Postoperative deep wound infections in adults after spinal fusion: management with vacuum-assisted wound closure. J Spinal Disord Tech 2005;18:14-17

8. Singh K, Samartzis D, Heller JG, An HS, Vaccaro AR. The management of complex soft tissue defects after spinal instrumentation. J Bone Joint Surg [Br]2006;88B:8-15

9. 0'Connor J, Kells A, Henry S, Scalea T. Vacuum-assisted closure for the treatment of complex chest wounds. Ann Thorac Surg 2005;79:1196-200.

10. Picada R, Winter RB, Lonstein JE, et al. Postoperative deep wound infection in adults after posterior lumbosacral spine fusion with instrumentation: incidence and management. J Spinal Disord 2000;13:42-5.

11. Lim MR, Lee JY, Vaccaro AR. Surgical infections in the traumatized spine. Clin Orthop 2006;444:114-19

12. Bütter A, Emran M, Al-Jazaeri A, Ouimet A. Vacuum-assisted closure for wound management in the pediatric population. J Pediatr Surg 2006;41:940-2.

13. Fang A, Hu SS, Endres N, Bradford DS. Risk factors for infection after spinal surgery. Spine 2005;30:1460-5.

14. Richards BS, Herring JA, Johnston CE, Birch JG, Roach JW. Treatment of adolescent idiopathic scoliosis using texas scottish rite hospital instrumentation. Spine 1999;19(Suppl):1598-605.

15. Richards BS. Delayed infections following posterior spinal instrumentation for the treatment of idiopathic scoliosis. J Bone Joint Surg [Am]1995;77-A:524-9.

16. Abbey DM, Turner DM, Warson JS, Wirth TC, Scalley RD. Treatment of postoperative wound infections following spinal fusion with instrumentation. J Spinal Disord 1995;8:278-83

17. Levi AD, Dickman CA, Sonntag VK. Management of postoperative infections after spinal instrumentation. J Neurosurg 1997;86:975-80.

18. Soultanis K, Mantelos G, Pagiatakis A, Soucacos PN. Late infection in patients with scoliosis treated with spinal instrumentation. Clin Orthop 2003:17:116-23. 